\title{
Hochwasserabflüsse in Österreich - das HORA-Projekt
}

\author{
Flood flows in Austria - the HORA (flood-risk zoning) project
}

\author{
von R. Merz¹, G. Blöschl' ${ }^{1}$ und G. Humer ${ }^{2}$
}

\section{Kurzfassung/Summary}

Ziel des HORA-Projektes war die Ausweisung von Überschwemmungsflächen für Hochwasserabflüsse der Jährlichkeiten $\mathrm{T}=30$, $\mathrm{T}=100$ und $\mathrm{T}=200$ für das gesamte österreichische Flussnetz im Maßstab 1:500000. Dies entspricht der Bestimmung von Hochwasserabflüssen an über 10000 Knoten. Der vorliegende Beitrag beschreibt die Gesamtkonzeption des hydrologischen Teiles des HORA-Projektes, die auf drei Grundprinzipen aufbaut: Kombination von verschiedenen Informationsquellen, wie z. B. Abflussdaten, Niederschlagsdaten, Abflussbeiwerten, Daten aus Nachbargebieten und historischen Hochwässern, um ein möglichst umfassendes Bild der der Bestimmung der Hochwasserabflüsse zu erhalten; Kombination von automatischen Methoden und ExpertInneneinschätzung, um möglichst effektiv lokale Besonderheiten zu erfassen; und die Einbindung der Hydrographischen Dienste, um so die Zuverlässigkeit und Akzeptanz der Ergebnisse zu erhöhen.

This paper presents the approach and the results of a study in which 30, 100 and 200 year return period flood discharges were estimated for $26000 \mathrm{~km}$ of Austrian streams, corresponding to more than 10000 nodes. Three guiding principles were adopted: Combination of various sources of information including flood peak samples, rainfall data, runoff coefficients and historical flood data in order to obtain a comprehensive assessment of the flood flows; Combination of automatic methods and manual assessments by hydrologists to allow speedy processing and to account for the local hydrological situation; and involvement of the Hydrographic Services to increase the accuracy and enhance the acceptance of results.

\section{Einleitung}

Nach dem Hochwasser 2002 wurde vom Bundesministerium für Land- und Forstwirtschaft, Umwelt und Wasserwirtschaft und der Versicherungswirtschaft das Projekt HOchwasserRisikoflächen Austria (HORA) (Stiefelmeyer und Hlatky, 2008) ins Leben gerufen. Ziel des HORA Projektes war es, Überschwemmungsgebiete für Hochwasserabflüsse $\left(\mathrm{HQ}_{\mathrm{T}}\right)$ der Jährlichkeiten $\mathrm{T}=30, \mathrm{~T}=100$ und $\mathrm{T}=200$ bezogen auf das im Maßstab 1:500 000 erfasste österreichische Flussnetz (ÖK 500) mit einer Gesamtlänge von ca. $26000 \mathrm{~km}$ auszuweisen. Die Ermittlung der Überschwemmungsgebiete gliederte sich in einen hydrologischen und in einen hydraulischen Teil. Der vorliegende Beitrag widmet sich den hydrologischen Arbeiten zur Ermittlung der Hochwasserabflüsse $\mathrm{HQ}_{\mathrm{T}}$. Aufgrund der großen Anzahl der zu ermittelten Hochwasserabflüsse und der großen hydrologischen Variabilität in Österreich wurde ein neues Konzept entwickelt, welches im vorliegenden Beitrag vorgestellt wird.

\section{Konzeption}

Entsprechend der Datenverfügbarkeit wurde die Vorgehensweise im Projekt HORA in drei Schritte (Abb. 1) gegliedert: (a) Datenerhebung und Preprocessing, (b) Ermittlung von Hochwasserabflüssen an Pegelstellen und (c) Ermittlung von Hochwasserabflüssen an Stellen ohne Pegel. Eine besondere Herausforderung bei der Bestimmung von flächendeckenden Hochwasserwahrscheinlichkeiten in Öster-

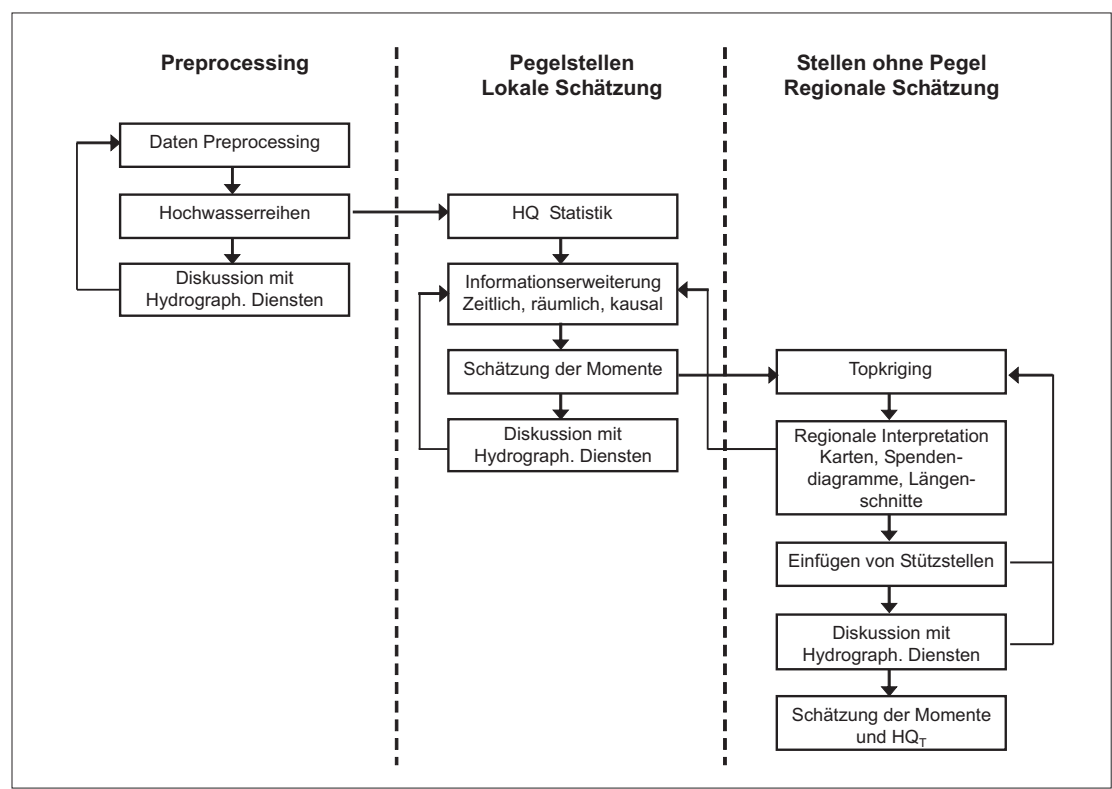

Abb. 1: Konzeption der Ermittlung von Hochwasserabflüssen 
reich ist die große Variabilität hydrologischer Prozesse. Ziel der hydrologischen Arbeiten war es, mit einem für ganz Österreich einheitlichen Verfahren, möglichst viele lokale Besonderheiten zu erfassen. Es wurde daher ein neues Konzept entwickelt, das auf drei Grundprinzipen aufbaut (Merz et al., 2008):

1) Kombination von verschiedenen Informationsquellen

2) Kombination von automatischen Methoden und Experteneinschätzung

3) Einbindung der Hydrographischen Dienste

\subsection{Kombination von verschie- denen Informationsquellen}

Traditionell beruht die Ermittlung von Hochwasserwahrscheinlichkeiten in Pegelgebieten auf einer rein statistischen Auswertung von beobachteten Hochwasserscheiteln. Plotting Positions, Wahl der Verteilungsfunktion, Methode der Parameterschätzung, Anpassungstests standen bisher im Mittelpunkt der Schätzung von Hochwasserwahrscheinlichkeiten (DVWK, 1999), während das umfangreiche Wissen der Ingenieurhydrologie über Prozesse und Zusammenhänge der Hochwasserentstehung bei der Berechnung nahezu nicht berücksichtigt wurde. Eine derartige Vorgehensweise setzt jedoch voraus, dass die Abflussbeobachtungen das Hochwasserverhalten des Gebiets vollkommen widerspiegeln. Dies ist jedoch nur selten der Fall. Die Abflussreihen sind in der Regel zu kurz, nicht stationär, fehlerbehaftet, entstammen nicht der gleichen Grundgesamtheit etc. Bei der Schätzung großer Jährlichkeiten muss in der Regel über den Bereich des bisher Beobachteten hinaus extrapoliert werden. Die Abflussprozesse bei Extremereignissen können sich jedoch von den üblicherweise beobachteten Hochwässern stark unterscheiden (Gutknecht, 1994). Im Hochwasser- kollektiv ist also in der Regel nicht genügend Information für eine zuverlässige Schätzung der Hochwasserwahrscheinlichkeiten enthalten. Die Schätzung von Hochwasserwahrscheinlichkeiten im Projekt HORA beschränkte sich deshalb nicht auf die statistische Auswertung von Abflussdaten, sondern verwendete auch Zusatzinformationen über das Hochwasserverhalten im betreffenden Gebiet. Diese Informationserweiterung kann daraus bestehen, die Abflussreihe in Bezug zur längeren hydrologischen Geschichte des Gebietes zu setzen, Informationen aus Nachbargebieten $\mathrm{zu}$ verwenden oder Prozesse der Hochwasserentstehung zu analysieren. Das Ziel war dabei, möglichst unterschiedliche Zusatzinformation im Hinblick auf Annahmen und Daten zu berücksichtigen, um ein vollständigeres Bild zu erhalten. Gedanklich folgt diese Vorgangsweise dem "Mehr-Standbeine“-Ansatz von Gutknecht et al. (2006), bei dem zur Berechnung von Bemessungshochwässer großer Jährlichkeiten lokale Hochwasserstatistik, regionale Hochwasserstatistik, NAModellierung, Gradex-Verfahren und Hüllkurven kombiniert werden.

Die Schätzung von Hochwasserwahrscheinlichkeiten an Stellen mit Abflussmessungen erfolgte im Projekt HORA dann durch eine Kombination von Hochwasserstatistik und Zusatzinformationen. Die Schätzwerte aus der Statistik und den zusätzlichen Informationen sind jeweils mit einem zugehörigen Vertrauensbereich oder Unschärfe-Bereich verbunden. In der klassischen Hochwasserstatistik entspricht dies den Konfidenzintervallen, oder dem Ergebnisspektrum bei Verwendung mehrerer Verteilungsfunktionen (DVWK, 1999). Die Größe des Unschärfe-Bereiches hängt von der Güte der verwendeten Daten und der Eignung und Güte des verwendeten Modells ab. Der maßgebende Hochwasserab- fluss wurde nun so gewählt, dass er möglichst den Schätzwerten aller Informationen entsprechend ihrer jeweiligen Unschärfebereiche entsprach. Für eine detaillierte Darstellung der Ermittlung von Hochwasserwahrscheinlichkeiten durch Kombination von Statistik und Informationserweiterung wird der Leser auf Merz und Blöschl (2008 $a, b, c)$ verwiesen.

Auch bei der Ermittlung von Hochwasserwahrscheinlichkeiten an Stellen ohne Pegel ist es sinnvoll, möglichst viele Informationen über das regionale Hochwasserverhalten in die Berechnung einfließen zu lassen. Natürlich stehen in diesem Fall weniger Informationen als bei Pegelgebieten zur Verfügung, jedoch können mehrere Methoden, die auf verschiedene Aspekte der Hochwasserentstehung eingehen, kombiniert werden, wie z.B. Informationen aus Nachbargebieten, Niederschlag-Abfluss Modellierung und Experteneinschätzung. Im HORA-Projekt wurden wiederum mehrere Informationsquellen herangezogen.

\subsection{Kombination von auto- matischen Methoden und Experteneinschätzung}

Viele der vorhandenen Zusatzinformationen können nicht leicht in eine quantitative Schätzung der Hochwasserkenngrößen umgewandelt werden, d.h. einzelnen Analysen der Zusatzinformationen resultieren nicht in einem automatischen Aufschlag oder Abschlag der Hochwasserkenngrößen um einen gewissen Prozentsatz. Manche Informationen erlauben nur qualitative Aussagen über ein plausibles Hochwasserverhaltenunderfordern ein gewisses Maß an ExpertInneneinschätzung. Gerade solche qualitativen Informationen beleuchten oft jene Aspekte des Hochwasserverhaltens, die in quantitativen, aus Messwerten abgeleiteten Aussagen nicht enthalten sind, jedoch für 
eine zuverlässige Bestimmung der t-jährlichen Hochwässer von entscheidender Bedeutung sind. So ist z. B. die Zuverlässigkeit der Abflussdaten ein wichtiger Einflussfaktor auf die Güte der Ergebnisse. Aufgrund der vielen unterschiedlichen Faktoren, die die Zuverlässigkeit der Abflussbeobachtungen bestimmen, wie z. B. die Extrapolation des Pegelschlüssels, Ausreißer, Messfehler, Umfließen des Pegels und kurze Beobachtungsreihen, die die gesamte Hochwasservariabilität nicht wiedergeben, ist eine formale Bestimmung, besonders auch für frühere Beobachtungen, kaum möglich. Jedoch sind bei den hydrographischen Landesdiensten qualitative Einschätzungen der Pegelgüte vorhanden, die im HORAProjekt in die Bestimmung von Hochwasserwahrscheinlichkeiten einflossen. Diese qualitativen Einschätzungen werden oft als „SoftData" bezeichnet. Neben der Datenzuverlässigkeit wurden weitere Aspekte der Hochwasserentstehung wie z. B. Ausuferungen, anthropogene Beeinflussungen und Rückschlüsse aus Morphologie und Gebietsbeschaffenheit auf das Hochwasserverhalten, durch qualitative Experteneinschätzungen bei der Bestimmung der Hochwasserkennwerten berücksichtigt. Auch betragen die Gebietsflächen vieler der über 10000 Gebiete, für die im HORA Projekt Hochwasserwahrscheinlichkeiten berechnetwurden, weniger als $50 \mathrm{~km}^{2}$. In diesen kleinen Einzugsgebieten können lokale Effekte, wie z. B. Abschattungen das Hochwasserverhalten dominieren (Gutknecht, 1994). Obwohl Österreich mit über 950 Pegeln ein sehr dichtes Durchflussmessnetz mit oft langen Beobachtungsreihen besitzt, können alle hydrologischen Besonderheiten dieser Gebiete nicht alleine durch beobachtete Hochwasserscheitel und deren statistischen Übertragung erfasst werden.

Die Bestimmung der Hochwasserwahrscheinlichkeiten in ein- zelnen Gebieten erfolgt daher in diesem Projekt durch eine iterative kombinierte Vorgangsweise von automatischen Vorschlagswerten und manueller Überprüfung. So wurde z. B. für die Pegelgebiete automatisch einzelne Vorschlagswerte für die Hochwasserwahrscheinlichkeitskurve aus der lokalen Statistik und quantitativer Zusatzinformation erzeugt und dann durch die SachbearbeiterInnen auf Basis von qualitativer Zusatzinformation kontrolliert und gegebenenfalls geändert. Für Gebiete ohne Pegel wurden Hochwasserabflüsse automatisch regionalisiert um dann wiederum auf Basis von qualitativer Zusatzinformation durch manuelles Setzen von Stützstellen auf lokale Besonderheiten einzugehen. Stützstellen sind Querschnitte in den die Hochwasserabflüsse durch die ExpertInnen vorgeben werden und an die sich die automatischen Regionalisierungsverfahren anpassen. Durch die Kombination von automatischen Verfahren und ExpertInneneinschätzung kann sehr effizient ein möglichst großes Maß an lokalen Besonderheiten bei der Bestimmung von Hochwasserwahrscheinlichkeiten in vielen Gebieten erfasst werden und dennoch bleibt ein größtmögliches Maß an Objektivität gewahrt.

\subsection{Einbindung der hydrogra- phischen Dienste}

Besonders viel Wissen über lokale hydrologische Besonderheiten ist bei den hydrographischen Diensten Österreichs vorhanden. Dieses Zusatzwissen kann von Informationen über historische Hochwässer, die lokale Hydrologie oder Einschätzung der Güte der hydrologischen Messdaten, vorangegangene Hochwasserstudien bis hin zu Erfahrungen aus Gebietsbegehungen reichen. Im Projekt HORA wurden daher die hydrographischen Landesdienste bei der Interpretation der Daten, Überlegungen zu wirksamen Pro- zessen und der daraus folgenden Schätzung der Hochwasserwahrscheinlichkeiten im Rahmen von Besprechungen eingebunden. Eine intensive Diskussion der lokalen Hydrologie von Gebieten bzw. Regionen erfolgte durch drei Besuche der einzelnen hydrographischen Dienste in drei verschiedenen Projektphasen. Die Aufgabenteilung wurde entsprechend Abb. 2 gesehen.

\section{Erhebung und Präprozes- sing der Daten}

Trotz der guten, digital verfügbaren Datenbasis von über 950 Pegeln war die Datenerhebung und -kontrolle ein sehr wichtiger Schritt des Projektes. Basis der Untersuchungen waren Reihen der maximalen jährlichen Abflussscheitel. Die Reihenlänge lag zwischen 5 und 180 Jahren mit einem Medien von ca. 35 Jahren. Die meisten Datensätze lagen auf Bundesebene vor. Um alle verfügbaren Quellen auszunützen, wurden zusätzliche Abflussdaten der Hydrographischen Landesdienste und der Kraftwerksgesellschaften erhoben und in den Datensatz integriert. Alle Datensätze wurden manuell auf Plausibilität durch Vergleich mit den Nachbarstationen geprüft. Dafür erwies sich die Diskussion mit den SachbearbeiterInnen der jeweiligen Landesdienste als besonders wertvoll. Die Datenqualität wurde für jede Station in einheitlicher Weise eingeschätzt und als Indikatorwert in den Datensatz aufgenommen. Ein kleiner Teil der Werte wurde korrigiert, und einige Stationen wurden aus der weiteren Bearbeitung ausgeschlossen. Etwaige Beeinflussungen durch wasserwirtschaftliche Maßnahmen wurden ebenfalls in die Beschreibung aufgenommen. Außerdem wurden auffällige Höchstwerte auf ihre hydrologische Entstehung hin untersucht. Einige der größten Durchflüsse waren Dammbruchwellen oder eine Fol- 




Abb. 2: Abstimmung mit den Hydrographischen Landesdiensten

ge von Verklausungen und wurden deshalb gesondert behandelt, da im Projekt die Hochwasserabflüsse zufolge hydrologischer Ursachen zu berechnen waren. Bei einigen Stationen war es zu Stationsverlegungen gekommen, die dazu führten, dass zwei Datenreihen in unmittelbarer Nachbarschaft vorlagen. Solche Reihen wurden kombiniert. Das Ergebnis dieses ersten Schrittes war ein nach einer einheitlichen Vorgangsweise kontrollierter und möglichst umfangreicher Datensatz der Jahreshöchstabflüsse in Österreich einschließlich einer Beschreibung der Datenqualität in Hinblick auf die Zuverlässigkeit der Durchflusswerte. Zahlreiche weitere Informationen betreffend die Hydrologie und Wasserwirtschaft der Gebiete wurden in ein geographisches Informationssystem integriert.

\section{Ermittlung der Hochwas- serwahrscheinlichkeiten für Pegelstellen}

Die Bestimmung der Hochwasserabflüsse an den Pegelstellen erfolgte durch die Kombination der Schätzwerte aus Statistik und den Zusatzinformationen. Eine Art der Zusatzinformation ist die längere hydrologische Geschichte des Gebietes. Oft treten große Hochwässer innerhalb weniger Jahre auf, wie z. B. 2002 und 2005, gefolgt von
Jahren ohne große Hochwässer. Erstreckt sich eine Beobachtungsreihe nur über einen Zeitraum mit wenigen bzw. sehr vielen Extrem-Jahren, so spiegelt die Beobachtungsreihe nicht die Grundgesamtheit wider, und daraus abgeleitete Hochwasserkenngrößen können das Hochwasserverhalten über- bzw. unterschätzen. Im HORA-Projekt wurden daher Gebiete mit Nachbargebieten verglichen, die längere Reihen aufwiesen. Es wurde hierbei eingeschätzt, wie sich das kurze Zeitfenster in das längere Bild einordnet. Für diesen Arbeitschritt liegen zwar formalisierte Methoden vor, wie z. B. Methoden zur Adjustierung des mittleren jährlichen Hochwassers aufgrund Klimavariabilität (IH, 1999), es erwies sich aber effizienter, diese Einschätzung nicht formalisiert, sondern durch ExpertInnenbeurteilung durchzuführen (Merz und Blöschl, 2003). Falls vorhanden wurden historische Hochwässer in die Untersuchung aufgenommen (Bayliss und Reed, 2001). In einigen Landesdiensten lagen umfangreiche Analysen historischer Hochwässer vor. Auch Fotografien historischer Hochwässer wurden herangezogen, um näherungsweise das Ausmaß der Überschwemmung einzuschätzen und damit eine Reihung der Jährlichkeiten historischer Hochwässer und der größten Hochwässer des Kollektivs zu ermöglichen. Die Informationen aus historischen Hochwässern erwiesen sich besonders im Hinblick auf die Extrapolation zu höheren Jährlichkeiten als eine sehr wertvolle Hilfe.

Eine weiter Art der Zusatzinformation sind Informationen aus Nachbargebieten. Im HORA-Projekt wurde deshalb Österreich unter Berücksichtigung topographischer, hydrogeologischer und klimatologischer Verhältnisse in 28 Teilregionen unterteilt und für jede Region die Abflussspende der Jährlichkeit $\mathrm{T}=100$ aus der Pegelstatistik gegen die Fläche aufgetragen (Spenden- diagramme). Auch mit der TopKriging Methode (siehe Abschnitt 5) regionalisierte Abflussspenden (ohne Verwendung des lokalen Wertes) wurden in die Spendendiagramme eingetragen. Mit Hilfe dieser Abflussspendendiagramme und Karten der Spenden, in denen auch Zusatzinformationen (z. B. der Jahresniederschlag) eingetragen waren, erfolgte ein manueller regionaler Abgleich der statistischen Momente (Mittelwert, Variationskoeffizient und Schiefe). Die Einschätzung der Zuverlässigkeit des betreffenden Pegels im Vergleich zur Zuverlässigkeit der Nachbarpegel war ein wichtiger Aspekt bei dem regionalen Vergleich.

Eine dritte Art von Zusatzinformation wurde aus der Analyse der Hochwasserprozesse gewonnen. Das Niederschlagsverhalten wurde unter anderem mit Hilfe des Gradex-Verfahrens berücksichtigt. Wegen der einfachen Struktur und des geringen Datenbedarfes ist das Gradex-Verfahren auch für eine regionale Anwendung geeignet (Merz et al., 1999). Da das GradexVerfahren die meist wesentlich längeren Reihen des Niederschlages einbezieht, war diese Zusatzinformation vor allem bei kurzen bzw. unsicheren Abflussreihen von Interesse, insbesondere für Gebiete mit einer Reaktionszeit um einen Tag, da Tageswerte des Niederschlages für das Gradex-Verfahren herangezogen wurden. Weiterhin wurden Abflussbeiwerte von Hochwässern aus den Untersuchungen von Merz et al. (2006) verwendet. Abb. 3 zeigt beispielhaft die empirischen Hochwasserwahrscheinlichkeiten und die dazugehörige Abflussbeiwerte für das niederschlagsreiche Gebiet Zwing an der Weißach (Vorarlberg) und das vergleichsweise niederschlagsarme Gebiet Fahrafeld an der Triesting (Niederösterreich). Im Zwing Gebiet sind die Abflussbeiwerte der Hochwässer in der Regel sehr hoch. Diese deuten auf eine stetig hohe Abflussbereitschaft des 
Gebietes hin. Große Hochwässer unterscheiden sich deshalb von kleineren Hochwässern nur durch die Größe des Niederschlages. Es ist somit eine eher kleine Schiefe der Hochwasserwahrscheinlichkeitsverteilung $\mathrm{zu}$ erwarten (Abb. 3a). Im Fahrafeld Gebiet sind die Abflussbeiwerte meistens klein, steigen jedoch mit der Größe des Hochwassers an. Große Hochwässer unterscheiden sich deshalb von kleineren Hochwässern nicht nur durch die Größe des Niederschlages, sondern auch durch einen wesentlich größeren Abflussbeiwert. Für solche Gebiete ist somit mit einer wesentlich größeren Schiefe der Hochwasserwahrscheinlichkeitskurve zu rechnen. Derartige Überlegungen wurden insbesondere für die Einschätzung der Schiefe an den Pegelstellen herangezogen.

Im Bedarfsfall wurden die Hochwasserwellen in stündlicher Auflösung visuell dargestellt um damit die Größe und Zuverlässigkeit des Scheitelwertes einzelner Ereignisse einzuschätzen. Im Vergleich mit Nachbargebieten konnte so die Abflussreaktion (rasch bzw. gedämpft) beurteilt werden um daraus Rückschlüsse auf die Scheitelwerte zu ziehen. Ebenso wurde die Prozessklassifikation von Merz und Blöschl (2003) herangezogen, die über 11000 Hochwasserereignisse in Hinblick auf ihre Entstehungsmechanismen klassifiziert. Bei Einzugsgebieten mit häufigen Gewittern etwa ist eine große Schiefe $\mathrm{zu}$ erwarten, bei Einzugsgebieten mit einem großen Anteil an Schneeschmelzhochwässern sind eher kleine Variationskoeffizienten und kleine Schiefen zu erwarten.

Eine wichtige Zusatzinformation war die Einschätzungen des hydrologischen Verhaltens auf Basis persönlicher Gebietskenntnis durch Begehungen und von Detailstudien. Beispielsweise können Morphologie und Bewuchs Informationen über das hydrologische Verhalten eines Gebietes geben
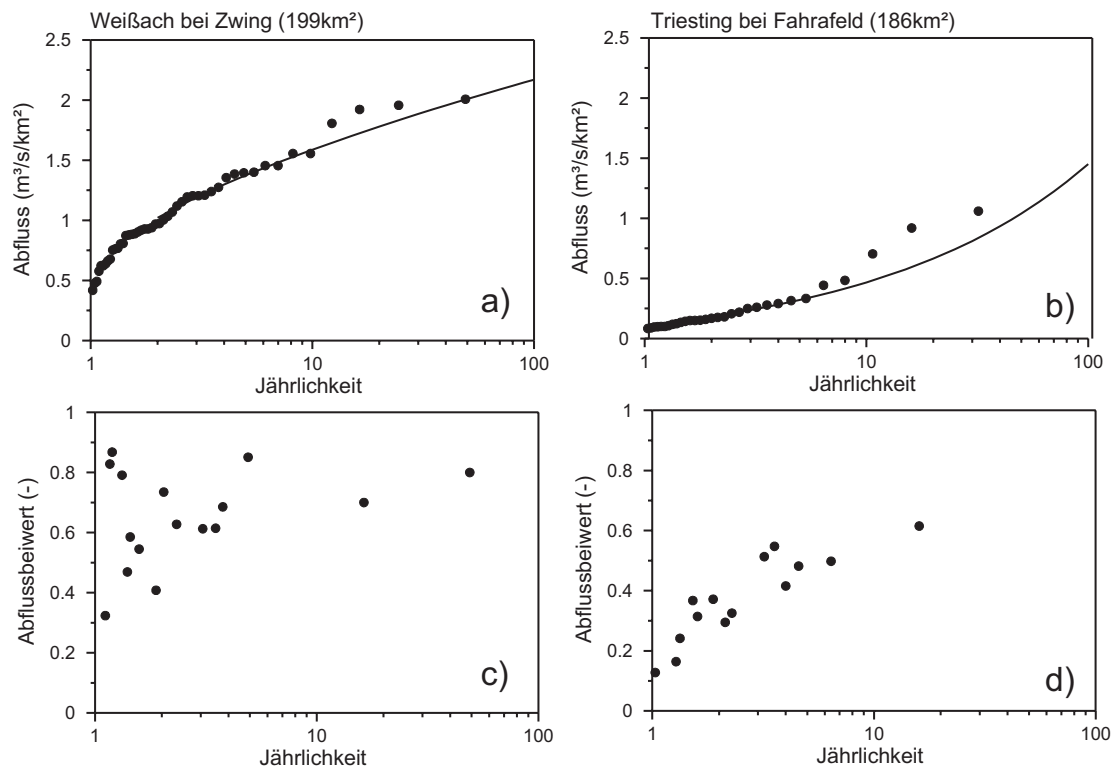

Abb. 3: Hochwasserwahrscheinlichkeiten (a, b) sowie Abflussbeiwerte der jeweiligen Ereignisse (c, d) aufgetragen gegen die Jährlichkeiten der Hochwasserscheitel. (a, c) Weißach bei Zwing $\left(199 \mathrm{~km}^{2}\right)$; (b, d) Triesting bei Fahrafeld $\left(186 \mathrm{~km}^{2}\right)$

(Aulitzky, 1992). Ein bemooster Gewässerlauf deutet eher auflangsame Abflussreaktionen und damit kleine Hochwasserspenden hin, während Erosionstätigkeit auf schnelle Abflussreaktionen und damit große Hochwasserspenden schließen lässt. Zwar waren aufgrund der Größe des Projektes persönliche Gebietsbegehungen durch die HORA SachbearbeiterInnen nicht möglich, die vorhandene lokale Gebietskenntnis bei den jeweiligen hydrographischen Landesdiensten wurde jedoch berücksichtigt. Auch digitale Karten der Geologie, des Boden, der Landnutzung, der Topographie, des Klima und anthropogenen Eingriffen waren eine wertvolle Hilfe bei der ExpertInneneinschätzung des Hochwasserrisikos und der Beurteilung der Aussagekraft anderer Informationsquellen.

Durch die Kombination von Hochwasserstatistik und Zusatzinformationen wurden für alle Pegel die Momente (Mittleres jährliches Hochwasser, Variationskoeffizient und Schiefe) ermittelt und danach Hochwasserwahrscheinlichkeiten unter Verwendung der allgemeinen Extremwertverteilung (AEV) be- rechnet. Die AEV-Verteilung ist flexibel genug, unterschiedliches lokales Hochwasserverhalten zu beschreiben und für die österreichischen Hochwasserkollektive durchwegs geeignet (Merz und Blöschl, 2005). Die manuell ermittelten Hochwasserwahrscheinlichkeitskurven aller Pegel wurden abschließend mit den hydrographischen Landesdiensten diskutiert. Bei Abweichungen zwischen den HORA-Werten und den zuvor bei den Landesdiensten vorliegenden Werten wurde der Grund der Abweichungen überprüft. Hatten die Landesdienste umfangreichere Informationen (z. B. Detailgutachten) als HORA verwendet, wurden diese bei HORA berücksichtigt. Waren die Unterschiede eine Folge der unterschiedlichen Einschätzung der Unsicherheiten, wurden die HORA Werte wegen der für Österreich einheitlichen Vorgangsweise belassen. An allen Pegeln konnten in der Diskussion Hochwasserwerte gefunden werden, welche den Einschätzungen der Landesdienste entsprachen bzw. ähnlich waren oder deren Unterschiede Folge einer unterschiedlichen Berücksichtigung der Unsicherheiten waren. 


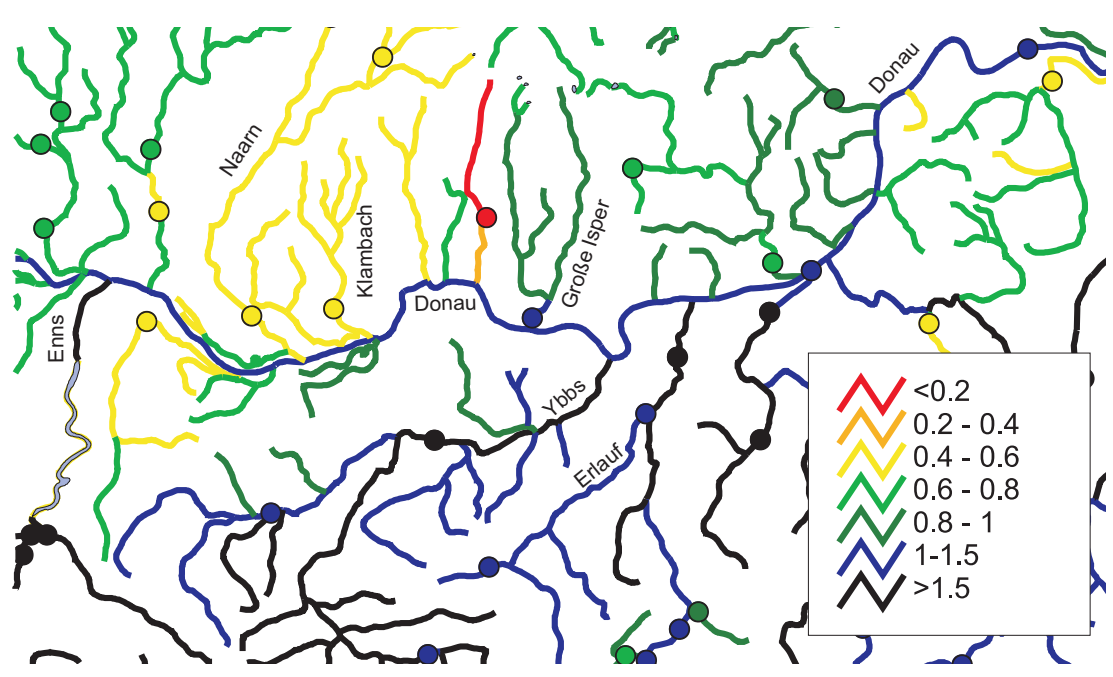

Abb. 4: Hundertjährliche Hochwasserspende im Donaugebiet zwischen Enns und Melk, normiert auf eine Gebietfläche von $100 \mathrm{~km}^{2}$ nach GI. 1. Kreise: Messwerte an den Pegeln. Linien: mittels Top-Kriging regionalisierte Werte

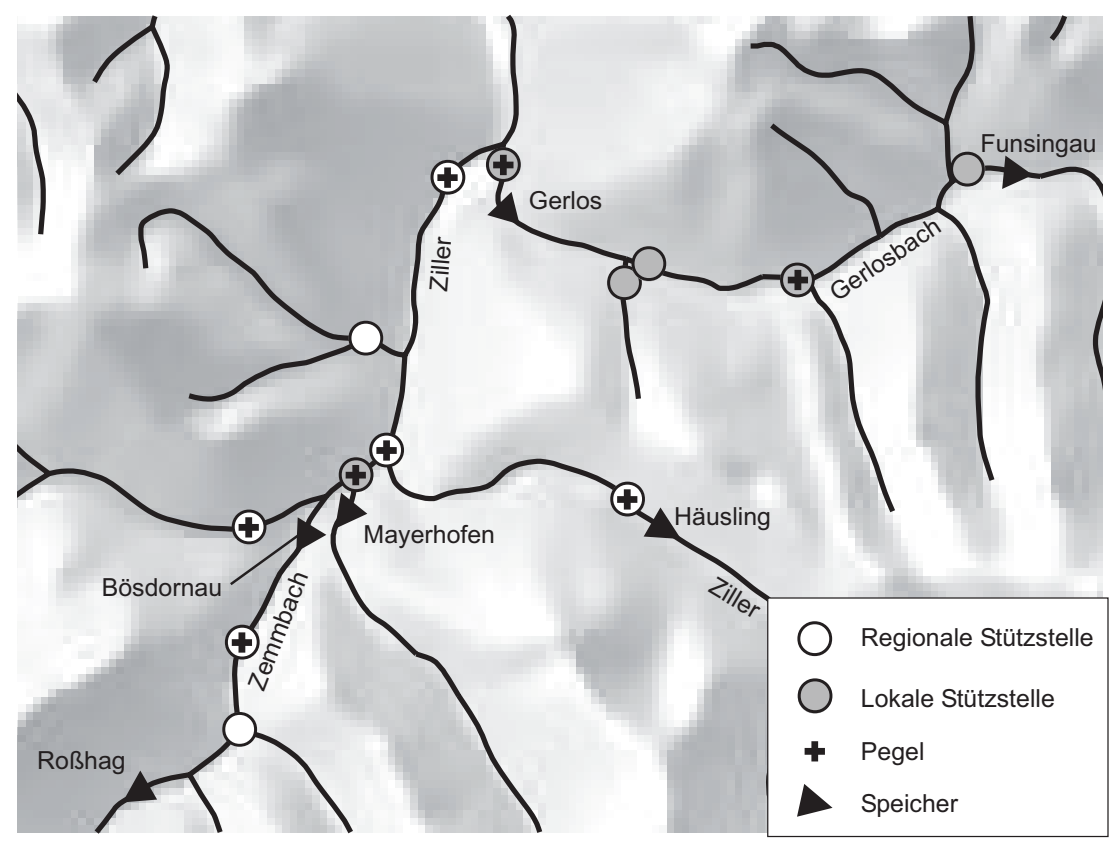

Abb. 5: Regionale und lokale Stützstellen der Ziller

\section{Ermittlung der Hochwas- serwahrscheinlichkeiten für Knoten ohne Pegel}

Für etwa 10500 Querschnitte ohne Abflussbeobachtungen wurden im HORA Projekt Hochwasserwahrscheinlichkeiten geschätzt. Ähnlich der Ermittlung der Hochwasserkenngrößen an Pegelstellen wurden die Hochwassermomente (Mittleres jährliches Hochwasser,
Variationskoeffizient und Schiefe) regionalisiert und dann unter Verwendung der AEV-Verteilung die Hochwasserwahrscheinlichkeiten für jeden Knoten ermittelt. Die Hochwasserkenngrößen sind von verschiedenen Einflussgrößen bestimmt, z. B. der Fläche, dem räumlichen hydrologischen Verhalten, dem Niederschlagverhalten, der Retention aufgrund von Seen und Speichern, dem menschlichen
Ausbau und Bewirtschaftungsmaßnahmen und lokalen hydrologischen Besonderheiten. Die Haupteinflussgrößen flossen wie folgt in die Berechnung ein:

Die Hochwasserspenden bestimmter Jährlichkeit nehmen in der Regel mit der Einzugsgebietsfläche wegen der meist räumlich ungleichmäßigen Überregnung ab. Um die Hochwasserspenden unterschiedlicher Einzugsgebietsflächen vergleichbar zu machen, wurde für die automatische Regionalisierung das mittlere jährliche Hochwasser auf eine nominale Größe von 100 $\mathrm{km}^{2}$ normiert mit

$$
M H q_{N}=A^{\alpha} \cdot 100^{-\alpha} \cdot M H q
$$

wobei $\mathrm{MHq}_{\mathrm{N}}$ die normierte mittlere jährliche Hochwasserspende für eine Bezugsfläche von $100 \mathrm{~km}^{2}$, Mhq die mittlere jährliche Hochwasserspende für die Gebietsfläche A und a der Exponent der Flächenreduktion ist. Dieser Exponent wurde getrennt für österreichische Regionen aus den Abflussdaten bestimmt und liegt zwischen 0.25 und 0.4 mit einem Mittelwert von 0.33. Im Staubereich der Alpen ist wegen der häufig räumlich gleichmäßigen Überregung der Exponent a klein (0.25), in Regionen mit häufigen konvektiven Niederschlägen ist $\alpha$ groß (0.4).

In Merz und Blöschl (2005) wurden verschiedene Regionalisierungsverfahren zur Bestimmung von Hochwasserkennwerten für Österreichische Verhältnisse verglichen, wobei geo-statistische Verfahren die besten Ergebnisse erzielten. Im HORA Projekt wurde daher ein geo-statistisches Verfahren, Top-Kriging (Skøien et al., 2006), angewandt, das im Gegensatz zum traditionellen Kriging die Topologie der Einzugsgebiete (Ober-, Unterlieger) berücksichtigt. Stellen am gleichen Flusslauf werden dabei als ähnlicher angesehen, als Stellen die nicht am gleichen Flusslauf liegen, auch wenn sie die 
gleiche räumliche Distanz aufweisen. Als Beispiel zeigt Abb. 4 die mit Topkriging berechnete 100 jährliche Hochwasserspende der Donauregion zwischen Enns- und Melkzufluß. Die große Variabilität in den beobachteten Abflüssen, mit kleineren Spenden an den Pegeln der nördlichen Zubringer und größeren Spenden im Süden werden durch Top-Kriging plausibel in die Fläche übertragen. Im Gegensatz $\mathrm{zu}$ anderen auf der räumlichen Distanz basierenden Regionalisierungsverfahren, sind z. B. die Abflüsse der Donau nicht durch diese kleinen Zubringer beeinflusst.

Der Einfluss der Reihenlänge der Hochwasserkollektive auf die Regionalisierung wurde, dem Vorschlag von Merz und Blöschl (2005) folgend, in Top-Kriging explizit durch die Kriging Varianz berücksichtigt. Somit konnten auch relativ kurze Reihen sinnvoll für die Regionalisierung der Hochwassermomente herangezogen werden.

In Österreich sind Hochwasserkenngrößen eng mit dem mittleren Jahresniederschlag korreliert, der ein Indikator für die Abflussbereitschaft von Gebieten ist (Merz, et al., 2006). Daher wurde der mittlere jährliche Gebietsniederschlag unter Berücksichtigung der Seehöhe für alle Gebiete ermittelt und die Mittelwerte der Hochwasserverteilung (MHq) mit Hilfe einer GeoRegression mit dem Niederschlag korreliert und mit Top-Kriging kombiniert.

Der Einfluss von Seen und wasserwirtschaftlichen Speichern auf die Hochwasserabflüsse wurde mit Hilfe eines Retentionsindex berücksichtigt, welcher eine österreichische Adaption des englischen Flood Attenuation by Reservoirs and Lakes (FARL) Index (IH, 1999) ist und sich aus der Seefläche, der Einzugsgebietsfläche des Sees und der Fläche des gesamten Einzugsgebietes errechnet. Neben den natürlichen Seen prägen menschliche Ausbau- und Bewirtschaf- tungsmaßnahmen das Hochwasserverhalten vieler Gebiete. Die anthropogenen Einflüsse wurden im HORA-Projekt für jedes Gebiet manuell unter Einschätzung der jeweils zutreffenden Situation berücksichtigt. Bemessungswerte für Talsperren und Hochwasserrückhaltebecken flossen dabei ein und Beileitungen wurden in Sinne eines „worst case“ den Hochwasserkennwerten zugeschlagen.

Weitere Einflussfaktoren der regionalen Hochwasservariabilität sind z. B. Topographie, Geologie, Landnutzung etc. Das Zusammenspiel dieser Einflussfaktoren bei der Entstehung von Hochwasserereignissen ist sehr komplex und deshalb durch formale Regionalisierungsmethoden wie Mehrfachregressionen kaum zu fassen (Merz und Blöschl, 2005). Die Berücksichtigung dieser Einflussfaktoren erfolgte daher im HORA-Projekt wieder durch ExpertInneneinschätzung.

Den Grundprinzipen des Projektes folgend wurden für jeden Flussabschnitt die Hochwassermomente durch das automatische Regionalisierungsverfahren geschätzt und anschließend manuell unter Berücksichtigung lokaler Besonderheiten überprüft und gegebenenfalls geändert. Bei der manuellen Einschätzung wurde die ÖK 50 sowie Karten der Topographie, Hydrogeologie, der Vegetaion und des Niederschlag verwendet. Spendendiagramme für einzelne Regionen dienten als wichtiges Werkzeug zur Überprüfung der Plausibilität der automatischen Schätzungen.

Die manuelle Einschätzung der lokalen Besonderheiten diente in einem iterativen Prozess als Eingangsgröße für die automatische Regionalisierung. Die Einbindung der manuellen Einschätzung erfolgte über die Zuweisung von zwei Typen von Stützstellen des automatischen Regionalisierungsverfahren. Der erste Typ sind regionale Stützstellen, d. h. Knoten, an denen eine lokale Schätzung der Hochwasserabflüsse vorliegt, die für die regionale Übertragung verwendet wird Dies sind einerseits Pegelstellen, die für die regionale Übertragung verwendet werden, aber auch Knoten an denen die Hochwasserkennwerte durch ExpertInneneinschätzung zugewiesen wurden. Dadurch ließen sich lokale Besonderheiten (z. B. Geologie) in die Regionalisierung einbringen. Der zweite Typ sind lokale Stützstellen, d. h. solche Knoten, an denen die Durchflusswerte nur lokal gültig sind, also nicht für die regionale Übertragung verwendet werden. Durch Kraftwerke beeinflusste Flussstrecken wurden beispielsweise in dieser Weise markiert. In Abb. 5 sind die Zuweisungen der lokalen und regionalen Stützstellen für die Ziller, aufgrund des Einflusses der Kraftwerke eingetragen.

Für Kopfgebiete, d.h. Gebiete am Beginn des Gewässers waren keine digitalen Gebietsgrenzen vorhanden. Für diese Gebiete wurden topographische Einzugsgebietsgrenzen durch Verschneidung des digitalen Geländemodells mit dem Gewässernetz generiert und damit die Hochwasserkennwerte berechnet.

\section{Ermittlung von Hochwas- serwahrscheinlichkeiten an großen Flüssen}

Für große Flüsse (Rhein, Inn, Salzach, Traun, Enns, Thaya, March, Mur, Drau und Donau) erfolgte die Bestimmung der Hochwasserkenngrößen mittels hydrologischer Längenschnitte.

Da in großen Flüssen das Hochwasserverhalten besonders durch die Verformung der Welle im Gerinne selbst bestimmt ist, wurde das Ausuferungsverhalten auf Basis der ÖK 50 und des digitalen Geländemodells abgeschätzt. Die Änderungen des Durchflusses entlang des Flusslaufes in den Längenschnitten wurden dann in Abstim- 




Abb. 6: Hundertjährliche Hochwasserspende $\left(\mathrm{m}^{3} / \mathrm{s} / \mathrm{km}^{2}\right)$

mung mit der Einschätzung des Ausuferungsverhaltens und der Zuflüsse der Zubringer gebracht.

\section{Ergebnisse und Diskussion}

Abbildung 6 zeigt die hundertjährliche Hochwasserspende in Österreich. Es zeigen sich große regionale Unterschiede. Die größten Abflussspenden sind am Alpennordrand zu erkennen. In dieser Region werden auch die höchsten Jahresniederschläge mit mehr als $2000 \mathrm{~mm}$ / Jahr gemessen, die vorwiegend auf Hebungsprozesse der von NordWest kommenden Luftmassen am Alpennordrand zurückzuführen sind. Da die Gebiete aufgrund der häufigen Niederschläge zu Ereignisbeginn sehr feucht sein können, kann die Kombination ausgiebiger Niederschläge mit hoher Abflussbereitschaft oft zu extremen Abflüssen führen. Eine Analyse der hochwasserauslösenden Prozesse (Merz und Blöschl, 2003) zeigte, dass fast alle Hochwässer in dieser Region auf Stauniederschläge zurückzuführen sind. Wesentlich kleinere Hoch- wasserspenden sind in den niederschlagsarmen Gebieten im Osten Österreichs zu erkennen. Mit 400 bis $500 \mathrm{~mm} / \mathrm{Jahr}$ beträgt der mittlere Jahresniederschlag weniger als ein Viertel des Niederschlages am Alpennordrand. Die Gebiete sind dadurch weniger feucht, die mittlere Abflussbereitschaft ist geringer (Merz et al., 2005) und auch die hochwasserauslösenden Prozesse sind unterschiedlich. In dieser Region führen eher kurzzeitige Niederschläge zu hohen Abflüssen.

Deutliche regionale Unterschiede sind auch in der Steigung der Hochwasserwahrscheinlichkeitskurve zu erkennen. Dies ist in Abb. 7 durch das Verhältnis des hundertjährlichen Abfluss zum mittleren jährlichen Hochwasserabfluss ausgedrückt. In den regenreichen Gebieten am Alpennordrand sind die hundertjährlichen Abflüsse nur zwei bis zweieinhalb mal so groß wie die mittleren jährlichen Hochwässer. Die trockenen Gebiete im Osten zeigen ein stärkeres nichtlineareres Verhalten der Hochwasserwahrscheinlichkeits- kurve. Die mittleren Hochwässer sind dort eher klein, aber es können, wenn auch selten, extreme Abflüsse auftreten. Entsprechend liegt das Verhältnis des 100 jährlichen Abflusses zum mittleren Jährlichen Hochwasserabfluss um 4 bis 5 .

Die regionalen Muster der Hochwasserkennwerte stimmen mit den Ergebnissen aus früheren methodisch ausgerichteten Analysen regionaler Hochwasserwahrscheinlichkeiten in Österreich (z. B. Merz und Blöschl, 2005) überein. Lokal können jedoch Unterschiede auftreten. Insgesamt zeigen die HORA Ergebnisse eine räumlich differenziertere und plausiblere Auswertung der Hochwasserwahrscheinlichkeiten. Durch die Erweiterung der Information über die beobachteten Hochwasserkollektive hinaus, konnten sehr viele hydrologische Besonderheiten erfasst werden. Durch die manuelle Einschätzung der Pegelgebiete und die manuelle Nachbearbeitung der Gebiete ohne Abflussmessungen konnten auch jene qualitativen Informationen über das Hochwasserverhalten in die Berech- 


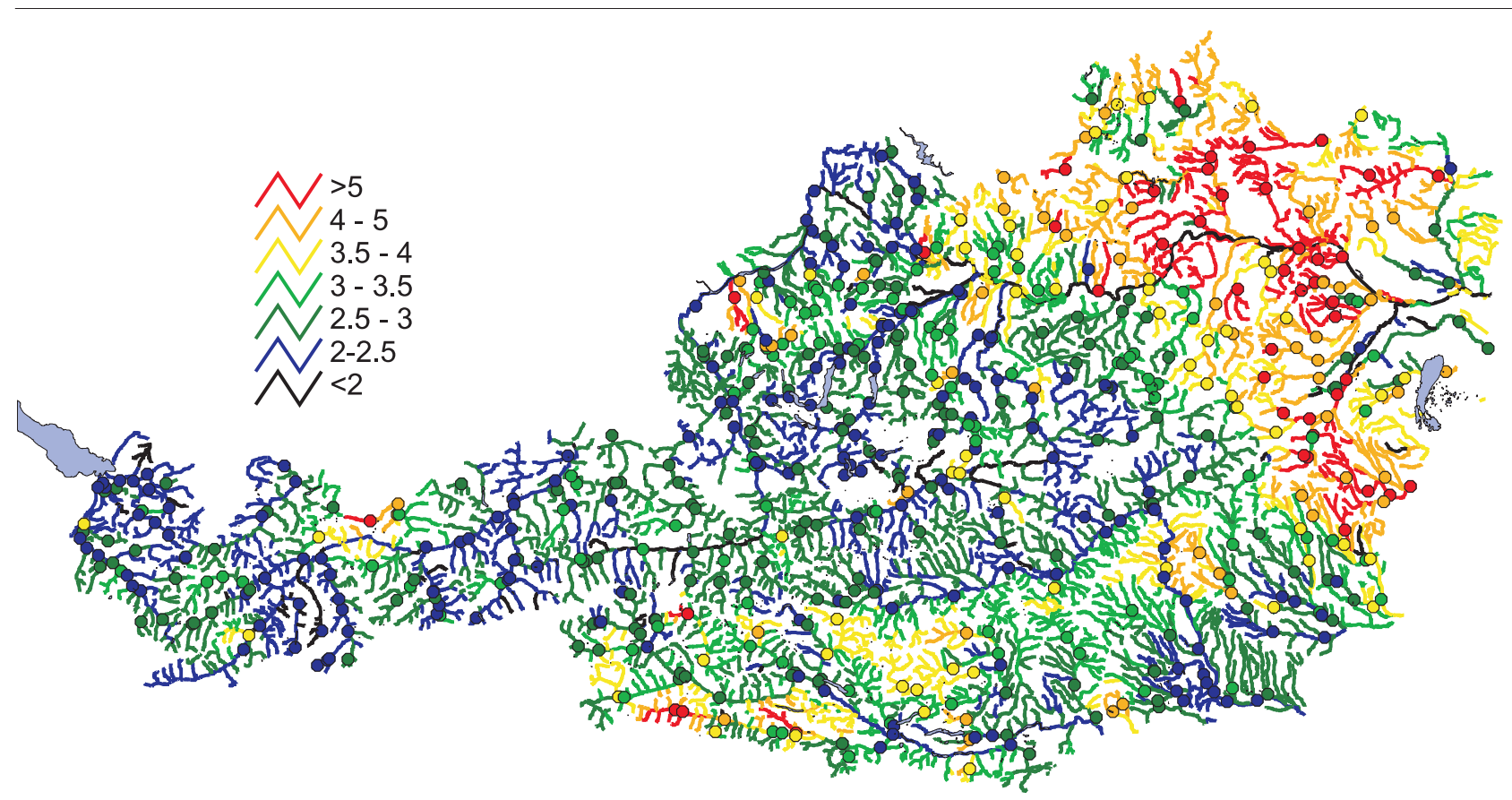

Abb. 7: Verhältnis von 100 jährlichem Abfluss zu mittlerem jährlichem Hochwasserabfluss

nung einfließen, die bei einer reinen Anwendung von automatischen Berechnungsverfahren nicht berücksichtigt werden können.

Mit Hilfe des Kombinationsansatzes von automatischen Vorschlagswerten mit manueller Einschätzung der SachbearbeiterInnen, konnte der Vorteil einer räumlich konsistenten Auswertung in Österreich mittels einer einheitlichen Methode mit der Einbeziehung von Informationen über lokale Besonderheiten verbunden werden. Der gewählte Kombinationsansatz erwies sich als sehr effizient. Dadurch war es möglich, innerhalb des Projektzeitraumes von 12 Monaten Hochwasserwahrscheinlichkeiten für über 10000 Gebiete plausibel zu schätzen.

Fallbeispiele von Vergleichen der HORA-Ergebnissen mit Detailstudien, z. B. Bemessungshochwasser für Talsperren, zeigen, dass im Projekt eine recht hohe Genauigkeit erzielt werden konnte. Allerdings kann bei einer solchen großmaßstäblichen Vorgangsweise nicht auf alle lokalen Besonderheiten einge- gangen werden, wie dies bei lokalen Gutachten (u. U. unter Verwendung von Niederschlag-Abflussmodellen) möglich ist. Deshalb sind die in diesem Projekt bestimmten Hochwasserwerte nicht als Bemessungswerte für lokale Fragestellungen anzusehen. Diese sollten weiterhin über Detailgutachten bestimmt werden, möglich unter Zusammenschau mit den HORA-Werten.

Ein wichtiger Schritt in der Ermittlung der Hochwasserwahrscheinlichkeiten war die Einbindung der hydrographischen Landesdienste. Durch Einbeziehung der Informationen über die Datenqualität und Unsicherheiten in den Abflussmessungen und die umfangsreiche Gebietskenntnis der SachbearbeiterInnen der hydrographischen Dienste konnte die Bestimmung der Hochwasserwahrscheinlichkeiten deutlich verbessert werden. Anderseits wurde auch eine höhere Akzeptanz der in HORA bestimmten Hochwasserabflüsse in den Ländern selbst, als auch bei Ingenieurbüros und anderen Stellen erreicht. Es wird vor- geschlagen, die HORA-Daten periodisch zu aktualisieren, um damit Informationen über jüngere Hochwässer einfließen zu lassen.

Die Ergebnisse der hydrologischen Regionalisierung im HORA-Projekt waren Eingangsgrößen in hydraulischen Berechnungen von Hochwasserrisikoflächen (Stiefelmeyer und Hlatky, 2008). Diese wurden vom Landwirtschaftlichen Rechenzentrum Wien zusammen mit Karten, Luftbildern und Adressdatenbanken in eine Internetapplikation (www.hochwasserrisiko.at) eingebunden. Mit Hilfe dieser Applikation ist es möglich, Luftbilder mit eingezeichneten Überflutungsflächen verschiedener Risikoklassen online per Adressabfrage $\mathrm{zu}$ erhalten. Die drei Risikoklassen entsprechen den in dem Projekt ermittelten Jährlichkeiten von 30 , 100 und 200 Jahren. Seit Juni 2006 ist die Internetapplikation auch für die Öffentlichkeit nutzbar. Das Projekt HORA stellt somit auch einen wichtigen Schritt zur Erfüllung der neu vorgelegten EU-Hochwasserrahmenrichtlinie (EU, 2006) dar. 


\section{Danksagung}

Für finanzielle Unterstützung wird der Österreichischen Akademie der Wissenschaften für ein APART [AUSTRIAN PROGRAMME FOR ADVANCED RESEARCH AND TECHNOLOGY] Stipendium und dem Bundesministerium für Landund Forstwirtschaft, Umwelt und Wasserwirtschaft Wien und dem Versicherungsverband Österreich
(Projekt: HOchwasserRisikozonierungAustria (HORA)) gedankt. Dem Hydrographischen Zentralbüro Wien und den Hydrographischen Landesdiensten Österreichs wird für die Bereitstellung der hydrologischen Basisdaten und die konstruktive Zusammenarbeit gedankt. Besonderer Dank gilt den Kollegen des Ingenieurbüros DI Günter Humer, (Geboltskirchen) für die $\mathrm{Zu}$ sammenarbeit im Projekt HORA.
Die Autoren sind O. Univ. Prof. Dr. h. c. D. Gutknecht für wertvolle Diskussionen zur Philosophie der prozessorientierten Modellierung zu Dank verpflichtet.

\section{Korrespondent:}

Institut für Wasserbau und Ingenieurhydrologie, TU Wien, Karlplatz 13/223, 1040 Wien

${ }^{2}$ Ingenieurbüro G. Humer, 4682 Geboltskirchen 70

\section{LITERATUR}

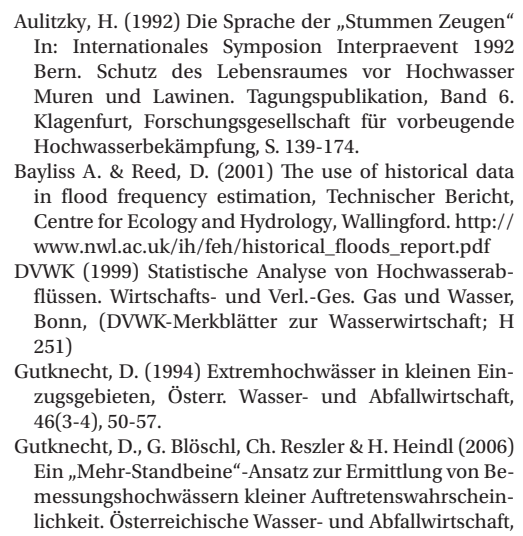

Gutknecht, D. (1994) Extremhochwässer in kleinen Einzugsgebieten, Österr. Wasser- und Abfallwirtschaft, 46(3-4), 50-57.

Gutknecht, D., G. Blöschl, Ch. Reszler \& H. Heindl (2006) Ein „Mehr-Standbeine“-Ansatz zur Ermittlung von Bemessungshochwässern kleiner Auftretenswahrscheinlichkeit. Österreichische Wasser- und Abfallwirtschaft,

58, H 3/4, pp. 44-50.

(1999) Flood Estimation Handbook, Institute of $\mathrm{Hy}-$ drology, Wallingford, UK.

kerz R \& Blöschl, G. (2003) A process typology of Merz R. \& Blöschl, G. (2003) A process typology of
regional floods. Water Resour. Res., 39(12), 1340, regional floods. Water Res

Merz R. und Blöschl G. (2008b) Flood Frequency Hydrology 1. Temporal, spatial and causal expansion
derz R. und Blöschl G. (2008b) Flood Frequency Hydrology 1. Temporal, spatial and causal expansio of information, Water Resc

Merz R. und Blöschl G. (2008c) Flood Frequency Hydrology 2. Combining data evidence, Water Resour. Res, 44, W08433, DOI 10.1029/2007 WR 006745

Merz R. und Blöschl, G. (2008a) Informationserweiterung zur Bestimmung von Hochwasserwahrscheinlichkeiten; Hydrologie und Wasserbewirtschaftung im Druck

Merz, R. and G. Blöschl (2005) Flood Frequency Regionalisation - spatial proximity vs. catchment attributes, Journal of Hydrology, 302 (1-4), 283-306.

Merz, R., Blöschl, G. \& Humer, G. (2008) National flood discharge mapping in Austria. Nat. Hazards. doi 10.10007/s11069-007 -9181-7.

Merz, R., Blöschl, G. \& Parajka, D. (2006) Raum-zeitliche Merz, R., Blöschl, G. \& Parajka, D. (2006) Raum-zeitliche
Variabilität von Ereignisabflussbeiwerten in Österreich, Hydrologie und Wasserbewirtschaftung, 50(1) S.2-11.

Merz, R., G. Blöschl und U. Piock-Ellena (1999a) Zur Anwendbarkeit des Gradex-Verfahrens in Österreich. Österr. Wasser- und Abfallwirtschaft, 51, Heft 11/12, pp. 291-305.

Skøien, J., Merz, R. \& Blöschl, G. (2006) Top-kriging - geostatistics on stream networks. Hydrology and Earth System Sciences, 10, 277-287.

Stiefelmeyer H. und Hlatky, Th. (2008) HORA - An Austrian Platform for Natural Hazards as a new Way in Ris Communication" In: Interpraevent 2008 Dornbirn. Schutz des Lebensraumes vor Hochwasser Muren und Lawinen. Tagungspublikation, Vol. 1. Klagenfurt, International Research Society INTERPRAEVENT, S. $229-236$ 